Stohmann: Calorimetrische Untersuchungen. 407 2 Atome Selen enthalten sind. Ihre Bildung würde sich gemüss der Gleichung:

$$
2 \mathrm{SO}_{2}+2 \mathrm{SeO}_{2}+2 \mathrm{H}_{2} \mathrm{O}=\mathrm{H}_{2} \mathrm{SSe}_{2} \mathrm{O}_{6}+\mathrm{H}_{2} \mathrm{SO}_{4} \text {, }
$$

und die Zersetzung des Barytsalzes durch ammoniakalische Silberlösung gemäss der Gleichung:

vollziehen.

$\mathrm{BaSSe}_{2} \mathrm{O}_{6}+\mathrm{Ag}_{3} \mathrm{O} .2 \mathrm{NH}_{3}+\mathrm{H}_{2} \mathrm{O}=\mathrm{BaSO}_{4}+\mathrm{Ag}_{2} \mathrm{Se}$

$$
+\left(\mathrm{NH}_{4}\right)_{2} \mathrm{SeO}_{4}
$$

Ich bedaure, vorläufig nicht in der Lage zu sein, die zum Theil lückenhaften Angaben über die beiden Säüren zu vervollständigen, hoffe indess später wieder auf den Gegenstand zurückkommen zu können, ohne mir indess hiermit das Gebiet reserviren zu wollen. Es verdiente übrigens versucht zu werden, ob nicht durch Einwirkung von schwefliger Süure auf Selensäure gleichfalls selen- und schwefelhaltige Säuren entstehen.

Santiago de Chile, Juli 1885.

\title{
Calorimetrische Untersuchungen;
}

von

F. Stohmann.

Vierte Abhandlung:

Ueber den Wärmewerth der Säuren der Fettsäurereihe; von

F. Stohmann und P. Rodatz.

Bei unseren bisherigen Untersuchungen über den Wärmewerth der Fettsäuren ${ }^{1}$ ) mussten wir uns auf die höheren Glieder dieser Reihe beschränken, weil die von uns benutzte Methode die Verbrennung von solchen Substanzen, bei welchen eine partielle Verflïchtigung bei gewöhnlicher Tempe-

1) Dies. Journ. [2] 31, 297; 32, 93. 
ratur zu erwarten ist, nicht zulässt. Da es aber sehr wünschenswerth erschien, auch den Wärmewerth der Anfangsglieder dieser Reihe kennen zu lernen, von denen seit Favre und Silbermann nur die Ameisensäure und die Essigsäure von Berthelot und von Thomsen, aber mit sehr abweichenden Resultaten, untersucht worden sind, so suchten wir nach einem Verfahren, um auch diese Körper unserer $\mathrm{Me}$ thode zugängig zu machen. Hierzu waren wir um so mehr veranlasst, da uns, aus später zu erörternden Gründen, die Richtigkeit der von Berthelot für die beiden Anfangsglieder der Fettsäurereihe gegebenen Zahlen zweifelhaft erscheinen musste.

Statt die freien Säuren zn verbrennen, gingen wir bei unseren Bestimmungen von den Silbersalzen aus. Durch Vergleichung des Wärmewerthes der Silbersalze mit dem direct ermittelten Wärmewerth der früher untersuchteri Säuren musste sich die Beziehung der sämmtlichen Säuren zu ihren Silbersalzen ergeben, und zu gleicher Zeit hätte die Differenz des Wärmewerthes der freien Säuren und der Silbersalze den Wärmewerth des durch Metalle vertretbaren Wasserstoffs des Carboxyls der Säuren geliefert. Leider zeigte sich bei der Ausführung, dass die Reaction nicht so glatt verläuft, wie wir angenommen hatten. Während die Silbersalze bei dem Verbrennen in freiem Sauerstoff geradeauf in Kohlensäure, Wasser und Metall zerfallen, findet beim Verbrennen mit Kaliumchlorat eine andere, nicht zu erwartende, Umsetzung statt. Das Silber wird dabei, bis auf Spuren, in Chlorsilber verwandelt, und ausserdem wird Kaliumcarbonat und Silicat, letzteres aus dem zugefügten Bimstein herrührend, gebildet. Bei der so leicht erfolgenden Umsetzung zwischen Chlorsilber und Kaliumcarbonat lässt sich das Nebeneinanderbestehen dieser beiden Verbindungen nur durch die Kürze der Reactionsdauer und durch die erhebliche Verdünnung der Masse des Kaliumcarbonats durch den grossen Ueberschuss des gleichzeitig entstehenden Chlorkaliums erklären.

Da weder die bei der Bildung des Chlorsilbers eintretende Wärmetönung, noch die bei der des Kaliumcarbonats 
Stohmann: Calorimetrische Untersuchungen. 409

für die hier obwaltenden Umstände bekannt ist und namentlich, da auch noch Kaliumsilicat entsteht, über dessen thermischen Werth wir nichts wissen, so musste auf eine rechnerische Correction verzichtet und eine solche auf empirischem Wege gesucht werden.

Ergab sich, bei der Vergleichung einer Anzahl von Säuren von bekanntem Wärmewerth und der gefundenen Verbrennungswärme der zugehörigen Silbersalze, eine constante, oder wenigstens innerhalb der Grenzen der Beobachtungsfehler liegende, gleichmässige Differenz, so musste diese Differenz die Wärmetönungen der Nebenprocesse, vermehrt durch den Wärmewerth des durch das Silber vertretenen Wasserstoffs der Carboxylgruppe, ausdrücken, und diese Zahl konnte, wenn sie übereinstimmend ausfiel, benutzt werden, um aus den für die Silbersalze ermittelten Werthen diejenigen der Säuren abzuleiten.

Wieweit eine solche Uebereinstimmung rorhanden ist, ergeben folgende Beobachtungen:

\begin{tabular}{|c|c|}
\hline Laurinsäure ${ }^{1}$ ) & 1747600 cal. \\
\hline Laurinsaures Silber & 1728717 \\
\hline Differenz & $18893 \mathrm{cal}$. \\
\hline Myristinsäure $^{1}$ ) & 2053824 cal. \\
\hline Myristinsaures Silber & 2034790 \\
\hline Differenz & 19034 cal. \\
\hline Benzoësäure ${ }^{2}$ ) & 766282 cal. \\
\hline Benzoësaures Silber & 746770 \\
\hline Differenz & 19512 cal. \\
\hline Salicylsäure ${ }^{2}$ ) & 712356 cal. \\
\hline Salicylsaures Silber & 694330 \\
\hline Differe & 18026 \\
\hline
\end{tabular}

Bei den hohen Molekulargewichten der betreffenden Verbindungen sind diese Differenzen so übereinstimmend, wie

1) Dies. Journ. [2] 32, 93.

") Das. [2] 31, 303. 


\section{Stobmann: Calorimetrische Untersuchuugen.}

möglich, und wir betrachten daher den sich daraus ergebenden Mittelwerth +18864 cal. (grösste Abweichung +648 und - 858) als Correctionszahl, um aus den für die Silbersalze gefundenen Werthen die Werthe für die Säuren im festen Zustande abzuleiten. Die Abweichungen von dieser Mittelzahl von +648 und -858 cal. können auf den ersten Anblick als zu beträchtlich erscheinen, als dass eine mit so grossen Fehlern behaftete Constante in die Rechnungen einzuführen erlaubt wäre. Berücksichtigt man aber, dass der niedrigste Werth, um welchen es sich hier handelt, der der Essigsäure, die Zahl von mehr als 200000 cal. erreicht, während der höchste, der der Myristinsäure, mehr als zehnmal so hoch ist, so ergiebt sich, dass die hierdurch bedingte Fehlergrösse niemals die Höhe von 0,4 Proc. übersteigen, ja bei der Myristinsäure nur 0,04 Proc. ausmachen kann. ${ }^{1}$ )

1) Bei der Beurtheilung der Zahlenwerthe für die Verbrennungswärmen ist nicht ausser Augen zu lassen, dass dieselben stets mit gewissen Beobachtungsfehlern behaftet sind, deren Grösse urngekehrt proportional der Menge der zu den Bestimmungen verwandten Substanz und direct proportional der Grösse des Molekulargewichts der Verbindung ist. Die Möglichkeit eine relativ grosse Menge von Substanz der Verbrennung zu unterziehen, ist daher ein grosser Vorzug unserer Methode. Berthelot (Ann. Chim. Phys. [5] 23, 153) schätzt die Genauigkeit der von ihm ausgefuhhrten Bestimmungen auf etwa 1 Proc. und sagt wörtlich: „Die Verbrennungswärmen vom Alkohol, Aethylen, Acetylen, Aethan liegen zwischen 300000 und 400000 eal. und sind daher mit einem wahrscheinlichen Fehler von 3 bis 4000 cal. behaftet; andererseits liegt die Verbrennungswärme des Benzols, des Diallyls und anuloger Körper zwischen 770000 und 900000 cal, der wahrscheinliche Fehlex wird daher bei diesen Körpern zwei- oder dreimal höher als bei jenen sein; die Verbrennungswärme der Zuckerarten ist annähernd $1400000 \mathrm{cal}$, der wahrscheinliche Fehler beträgt daher 14000 cal., die Verbrennungswärme der Stearinsäure ist doppelt so hoch als die der Zuckerarten, und damit verdoppelt sich auch der Beobachtungsfehler."

Wir haben von unseren Bestimmungen eine bessere Meinung als Berthelot von den seinigen, und betrachten die Fehlergrenze von einem Procent als zu hoch, namentlich seitdem unser Calorimeter dureh Beseitigung aller anderer Umhüllungen, als der der doppelten, abgegrenzten Luftschichten, deren Temperatur durch einen starken Wasserunantel coustant erhalten wird, wesentlich verbessert worden ist. 
Da die Säuren in den Silbersalzen im starren Zustande enthalten sind, so ist dem Verbrennungswerth noch die Schmelzwärme hinzuzurechnen, um den Wärmewerth der flüssigen Säuren zu erhalten. Die Schmelzwärmen sind aber nur für vier Säuren der ganzen Reihe bekannt, nämlich für Ameisensäure und Essigsäure, von Petterson ${ }^{1}$ ) bestimmt, und für Laurinsäure und Myristinsäure, welche von uns ${ }^{2}$ ) ermittelt wurden.

Dieselben betragen pro Gramm:

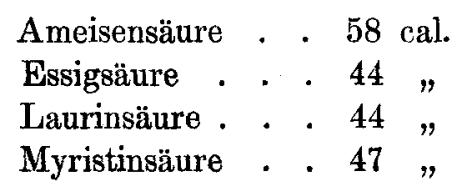

Bei dem weiten Abstande der beiden ersten und der beiden letzten Säuren zeigt sich hier keine nenneswerthe Verschiedenheit im Betrage der Schmelzwärme, es ist daher mit grösster Wahrscheinlichkeit anzunehmen, dass die Schmelzwärme sämmtlicher fetten Säuren, auf die Gewichtseinheit bezogen, gleich sei, und wir betrachten daher den Mittelwerth obiger Zahlen 48 cal. als Schmelzwärme für je ein Gramm sämmtlicher Säuren.

Um den Beweis hierfür zu liefern, greifen wir aus unseren neueren Bestimmungen willkürlich die der Laurinsäure heraus. Dieselbe ergab in vier Verbrennungen pro Molekül:

1748800 cal.
$1747400 "$
$1748600 "$
$1745800 "$

Setzt man hier den niedrigsten Werth $=100$, so werden die übrigen der Reihenfolge nach 100,18, 100,09, 100,16, und die Abweichung zwischen dem niedrigsten und dem höchsten Werth beträgt 3000 cal., während nach Berthelot eine Abweichung vom Mittelwerth von 17000 cal. zulässig sein würde.

Berthelot's Urtheil über unsere Methode: die Genauigkeit der mit vieler Mïhe und Sorgfalt erlangten Resultate bleibe weit von der beim Verbrennen mit freiem Sauerstoff erzielten entfernt, ist daher als unbegründet zurïckzuweisen.

1) Dies. Journ. [2] 24, 298.

2) Das. [2] 32, 80 . 


\section{Stohmann: Calorimetrische Untersuchungen.}

Wir haben demnach zwei Voraussetzungen zu machen, auf welchen die Brauchbarkeit unserer Untersuchung basirt. Erstens die Zulässigkeit unserer Correctionszahl für die Wärmetönungen der Nebenprocesse und zweitens die Richtigkeit der angenommenen Schmelzwärme. Dass diese beiden Prämissen nicht falsch sind, wird sich ergeben, wenn wir den aus den Silbersalzen abgeleiteten Wärmewerth mit dem durch directe Verbrennung der freien Säuren ermittelten vergleichen.

Von den Silbersalzen wurde das essigsaure, propionsaure, buttersaure und valeriansaure Silber, durch Zersetzung der entsprechenden Natriumsalze mit salpetersaurem Silber, in concentrirter Lösung gefällt und nach dem Auswaschen aus heissem Wasser umkrystallisirt. Die unlöslichen Salze der Capronsäure, Caprylsäure, Caprinsäure, Laurinsäure, Myristinsäure, Benzoësäure, Salicylsäure wurden aus schwach ammoniakalischer Lösung gefällt und mit schwachem Weingeist gewaschen. Alle Operationen geschahen im künstlich völlig verdunkelten Zimmer bei Gaslicht, um die Einwirkung des Sonnenlichtes auszuschliessen. Hierdurch gelang es, die Salze völlig unzersetzt und ungefärbt zu erhalten. Zur Constatirung der Reinheit wurden sämmtliche Silbersalze analysirt.

Essigsaures Silber. $1,108 \mathrm{Grm} .=0,7157 \mathrm{Grm} . \mathrm{Ag}=64,59$ Proc., berechnet 64,67 Proc.

Propionsaures Silber. $1,0809 \mathrm{Grm} .=0,6459 \mathrm{Grm} . \mathrm{Ag}=$ 59,76 Proc., berechnet 59,67 Proc.

Buttersaures Silber. $1,0855 \mathrm{Grm} .=0,5998 \mathrm{Grm} . \mathrm{Ag}=55,26$ Proc., berechnet 55,38 Proc.

Valoriansaures Silber. 1,216 Grm. $=0,630 \mathrm{Grm} . \mathrm{Ag}=51,86$ Proc., berechnet 51,68 Proc.

Capronsaures Silber, $1,262 \mathrm{Grm} .=0,5464 \mathrm{Grm} . \mathrm{Ag}=48,52$ Proc., berechnet 48,43 Proc.

Caprylsaures Silber. $\quad 0,9048$ Grm. $=0,3886$ Grm. $\mathrm{Ag}=42,95$ Proc., berechnet 43,03 Proc.

Caprinsaures Silber. $1,0445 \mathrm{Grm} .=0,4035 \mathrm{Grm} . \mathrm{Ag}=38,63$ Proc., berechnet 38,71 Proc.

Laurinsaures Silber, $0,9686 \mathrm{Grm} .=0,3398 \mathrm{Grm} . \mathrm{Ag}=35,09$ Proc., berechnet 35,18 Proc. 


\section{Stohmann: Calorimetrische Untersuchungen. 413}

Myristinsaures Silber. 1,0673 Grm. $=0,3438$ Grm. $\mathrm{Ag}=$ 32,21 Proc., berechnet 32,24 Proc.

Benzoësaures Silber. $1,0526 \mathrm{Grm} .=0,4968 \mathrm{Grm} . \mathrm{Ag}=47,20$ Proc., berechnet 47,16 Proc.

Salicylsaures Silber. $1,1193 \mathrm{Grm} .=0,4931 \mathrm{Grm} . \mathrm{Ag}=44,06$ Proc., berechnet 44,08 Proc.

Zur Verbrennung der Silbersalze diente unser ganz aus Platin gefertigtes Calorimeter IV. Bei den ersten Versuchen verwandten wir zur Aufnahme der Verbrennungsmischung die von Rubner empfohlenen Glashülsen, um einer eventuellen Zerstörung der Platinpatrone durch sich ausscheidendes metallisches Silber vorzubeugen. Nachdem wir aber gefunden hatten, dass metallisches Silber bei der Reaction nur in Spuren gebildet wird, und nachdem wir uns davon überzeugt hatten, dass die Glashülsen gegenüber unserer Platinpatrone nicht allein keinen Vortheil, sondern erhebliche Nachtheile besitzen, kehrten wir zu unserem bewährten Apparat zurück. ${ }^{1}$ )

1) Ueber den Werth der Glashülsen vergl, dies. Journ. [2] 31, 273 Anm. In einer neueren Publication (Zeitschr. f. Biologie 21, 258 ff.) kommt Rubner auf denselben Gegenstand zurück und sehreibt der Verwendung der Platinpatrone das Missglücken einer ganzen Reihe von Bestimmungen zu (l. c. S. 265). Wir müssen hiergegen Verwahrung einlegen, da dadurch die Brauchbarkeit unserer Methode beeinträchtigt erscheint. Rubner behauptet: „Bei manchen Substanzen kann man oft bemerken, dass durch das Zusammenschmelzen des Bimsteins (richtiger wohl durch die Bildung von Schlacken von geschmolzenem Chlorkalium) den entweichenden Gasen der Ausweg versperrt wird; dann erfolgt nach kurzem Stillestehen der Verbrennung plötzlich ein kräftiger Stoss, welcher nicht nur Bestandtheile der Verbrennungsmischung herausschleudert, sondern dieselbe geradezu unterbrechen kann." Diese stossweise Verbrennung hat aber mit der Verwendung der Platinpatrone nichts zu thun, sondern ist alle Male nur durch ein ungeeignetes Mischungsverhältniss der Materialien bedingt und lässt sich durch Abänderung dieses Verhältnisses vermeiden, wie wir bei der eingehenden Beschreibung der Methode (Landw. Jahrb. 13, 521) wiederholt hervorgehoben haben. Jede Verbrennung, bei welcher der geringste Stoss stattfindet, ist unbedingt zu verwerfen. Gegen die eintretende Spannung der Gase gewährt vielmehr die Beschaffenheit unserer Platinpatrone einen viel wirksarneren Schutz, als die Rub ner'sche Glashülse. Hat sich über dem nuverbrannten Theile der 


\section{Stohmann: Calorimetrische Untersuchungen.}

Die Oxydationsmischung bestand, wie in allen unseren Versuchen aus 13,33 Grm. Kaliumchlorat und 1,67 Grm. Braunstein, der vorher mit verdünnter Salpetersäure von allem darin Löslichen befreit und dann scharf geglüht war.

Zur Belebung der Verbrennung wurde bei den an Kohlenstoff ärmeren Salzen, bis zu dem der Valeriansäure, ein Zusatz von Paraftin von $74^{\circ}$ Schmelzpunkt gegeben, dessen Wärmewerth mit 11108 cal. pro Gramm in Rechnung gestellt wurde (s. dies. Journ. Bd. 31, 294). Als weiterer Zusatz diente krystallisirter Milchzucker, dessen Würmewerth 3663 cal. pro Gramm ist $(31,283)$. Von der Verwendung der Kerzenstearinsäure, deren wir uns sonst bei der Verbrennung von kohlenstoffarmen Substanzen bedienen, musste hier Abstand genommen werden, weil die Silbersalze durch die ätherische Lösung dieser Säure zersetzt werden. Essigsaures Silber liess beim Verreiben mit einer ätherischen Lösung von Stearinsäure auf das deutlichste den scharfen Geruch der Essigsäure wahrnehmen.

Der Wasserwerth des Calorimeters beträgt 29,4 cal. Die Wasserfüllung 2000 Grm.

Das Thermometer $8 \mathrm{~b}$ trägt eine willkührliche Scala von der je $100 \mathrm{pp}$. $=1,4384^{\circ}$.

Die allgemeine Correctionszahl ist jetzt bei galvanischer Zündung $=595$ cal. $(31,285)$.

Alle auf die Verbrennungen bezüglichen Daten finden

\footnotetext{
Mischung eine Schlacke gebildet, die den entweichenden Gasen den Ausweg versperrt, so entweichen diese bei unserem Apparat ohne jegliches Hinderniss durch die vier, in versehiedenen Höhen der Patrone angebrachten, seitlichen, Oeffnungen, welche in dem Masse, wie die Verbrennung fortschreitet, frei werden und also als Sicherheitsventile wirken. Das den Glashülsen nachgerühmte Abschmelzen haben wir nur ganz ausnahmsweise, das Aufbauchen des Glases und Platzen der Blase, durch welches die gespannten Gase entweichen, nie beobachtet. Dagegen bleibt die Glashülse in den meisten Fällen während der Verbrennung intact und zerfällt erst beim Zulassen des kalten Wassers. Aus der unverletzten Glashülse können die gespannten Gase aber nicht anders als gewaltiam, mit Stoss, sich einen Ausweg bahnen.
} 
Stohmann: Calorimetrische Untersuchungen. 415 sich in folgender Tabelle vereint. Hinsichtlich der Ableitung der Resultate verweisen wir auf die Beschreibung der Methode $\S 9^{1}$ ), wozu nur noch zu bemerken ist, dass gegenwärtig bei der galvanischen Zündung, wo die Anfangstemperatur erst abgelesen wird, nachdem völlige Temperaturausgleichung aller Theile des Apparates eingetreten ist, die dort mit $\mathrm{A}$ und $\mathrm{B}$ bezeichneten Correctionen für den Einfluss der umgebenden Luft in Wegfall kommen.

(Siehe Tabelle S. 416 und 417.)

Die Wärmewerthe der Silbersalze, pro Gramm-Molekül berechnet, ergeben sich demnach folgendermaassen:

Molekulargew. Wärmewerth

Essigsaures Silber $\quad \mathrm{C}_{2} \quad \mathrm{H}_{3} \quad \mathrm{Ag} \mathrm{O}_{2} \quad 167 \quad 191549 \mathrm{cal}$.

Propionsaures Silber $\mathrm{C}_{3} \mathrm{H}_{5} \mathrm{Ag} \mathrm{O}_{2} \quad 181 \quad 345529$

Buttersaures Silber $\mathrm{C}_{4} \mathrm{H}_{7} \mathrm{Ag} \mathrm{O}_{2} \quad 185 \quad 499590$

Valeriansaures Silber $\mathrm{C}_{5} \mathrm{H}_{9} \mathrm{Ag} \mathrm{O}_{2} \quad 209 \quad 652916$

Capronsaures Silber $\mathrm{C}_{6} \mathrm{H}_{11} \mathrm{Ag} \mathrm{O}_{2} \quad 223 \quad 806814$

Caprylsaures Silber $\quad \mathrm{C}_{8} \mathrm{H}_{15} \mathrm{Ag} \mathrm{O}_{2} \quad 251 \quad 1114189$

Caprinsaures Silber $\quad \mathrm{C}_{10} \mathrm{H}_{19} \mathrm{Ag} \mathrm{O}_{2} \quad 279 \quad 1422342$

Laurinsaures Silber $\mathrm{O}_{12} \mathrm{H}_{23} \mathrm{Ag} \mathrm{O} \mathrm{O}_{2} \quad 307 \quad 1728717$

Myristinsaures Silber $\mathrm{C}_{14} \mathrm{H}_{27} \mathbf{A g ~ O}_{2} \quad \mathbf{3 3 5} \quad 2034790$

Benzoësaures Silber $\mathrm{C}_{7} \mathrm{H}_{3} \mathrm{Ag} \mathrm{O}_{2} \quad 229 \quad \mathbf{7 4 6 7 7 0}$

Salicylsaures Silber $\quad \mathrm{C}_{7} \mathrm{H}_{5} \quad \mathrm{Ag} \mathrm{O}_{3} \quad 245 \quad 694330$

Das benzoësaure und das salicylsaure Silber interessiren uns hier nicht weiter, da die Bestimmungen des Wärmewerthes dieser Salze nur zu dem Zwecke ausgeführt sind, um Material für die Ermittelung der Zahl, welche die Beziehungen der für die Silbersalze gefundenen Werthe zu den gesuchten Werthen für die freien Süuren ausdrückt, zu gewinnen. Führt man die Rechnungen in der oben beschriebenen Weise aus, so ergeben sich für die flüssigen Fettsäuren die in der Tabelle S. 418 aufgeführten Wärmewerthe pro Gramm-Molekül:

1) Landw. Jahrb. 13, 538. 
416 Stohmann: Calorimetrische Untersuchungen.

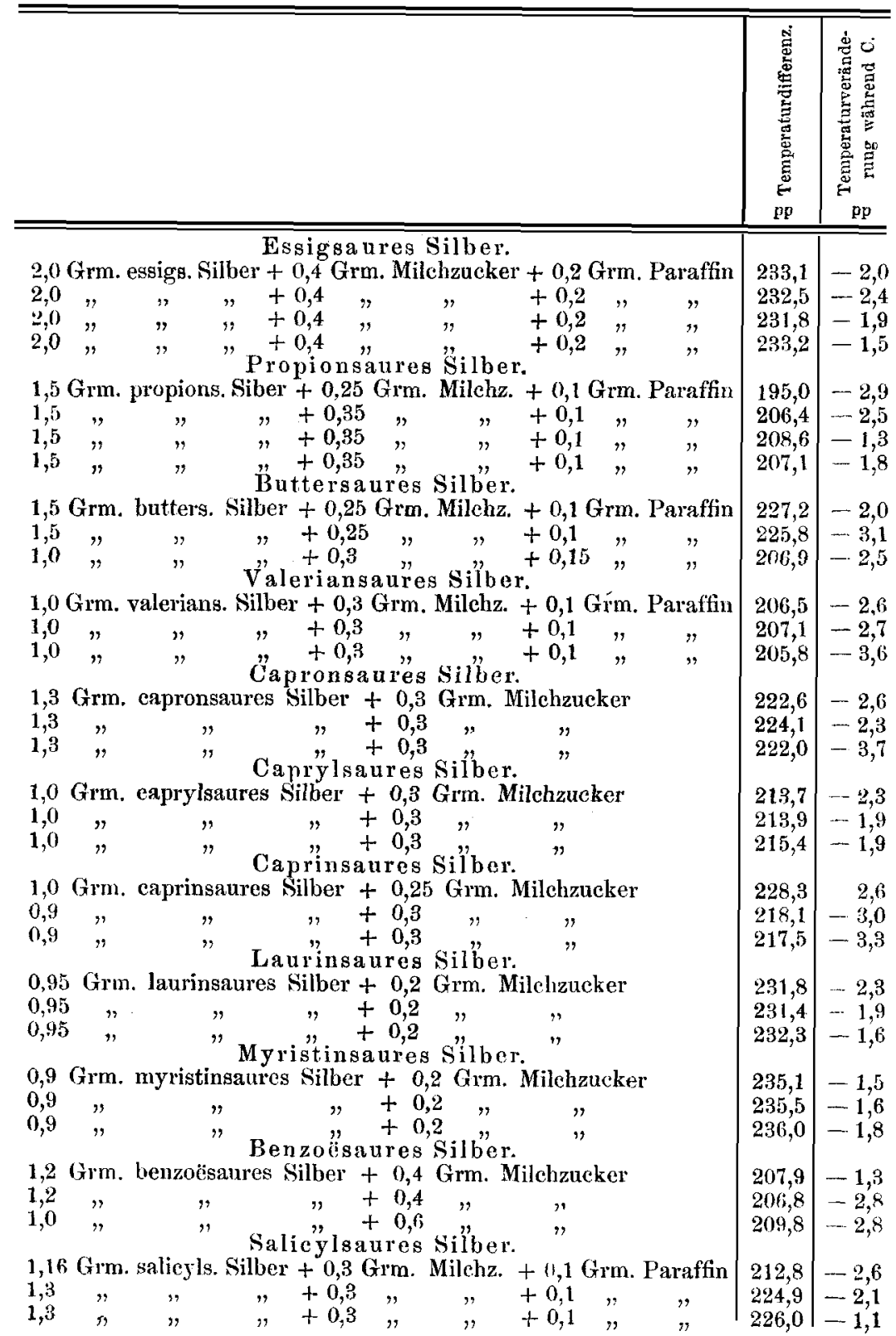


Stohmann: Calorimetrische Untersuchungen. 417

\begin{tabular}{|c|c|c|c|c|c|c|c|c|c|c|}
\hline $\begin{array}{r}\mathrm{Ti} \\
\text { Calor } \\
\text { Grm. }\end{array}$ & $\begin{array}{l}\text { irung d } \\
\text { neterwa } \\
\mathrm{Cl} \text { in } 5\end{array}$ & $\begin{array}{l}\text { Ccm. } \\
\text { Cers. }\end{array}$ & 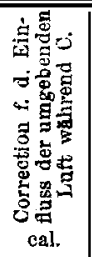 & 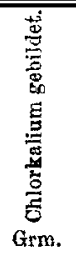 & 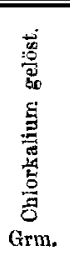 & 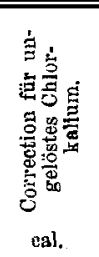 & 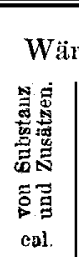 & 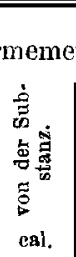 & 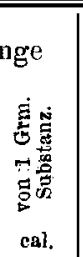 & $\begin{array}{l}\dot{0} \\
\pm \\
\pm \\
\equiv \\
\text { cal. }\end{array}$ \\
\hline $\begin{array}{l}0,0864 \\
0,1087 \\
0,0981 \\
0,0981\end{array}$ & $\begin{array}{l}0,1124 \\
0,1368 \\
0,1271 \\
0,1296\end{array}$ & $\begin{array}{l}0,1860 \\
0,1845 \\
0,1850 \\
0,1840\end{array}$ & $\begin{array}{l}-\quad 3 \\
-\quad 7 \\
-13 \\
-30\end{array}$ & $\begin{array}{l}7,27 \\
7,25 \\
7,25 \\
7,22\end{array}$ & $\begin{array}{l}3,46 \\
4,15 \\
3,92 \\
3,92\end{array}$ & $\begin{array}{r}-229 \\
-186 \\
-200 \\
-198\end{array}$ & $\begin{array}{l}5977 \\
5999 \\
5958 \\
5992\end{array}$ & $\begin{array}{l}2290 \\
2312 \\
2271 \\
2305\end{array}$ & $\begin{array}{l}1145 \\
1156 \\
1135 \\
1152\end{array}$ & 1147 \\
\hline $\begin{array}{l}0,0955 \\
0,1215 \\
0,1175 \\
0,1215\end{array}$ & $\begin{array}{l}0,1317 \\
0,1576 \\
0,1576 \\
0,1576\end{array}$ & $\begin{array}{l}0,1906 \\
0,1881 \\
0,1891 \\
0,1891\end{array}$ & $\begin{array}{r}0 \\
-12 \\
-56 \\
-32\end{array}$ & $\begin{array}{l}7,47 \\
7,43 \\
7,46 \\
7,48\end{array}$ & $\begin{array}{l}3,82 \\
4,86 \\
4,70 \\
4,86\end{array}$ & $\begin{array}{l}-219 \\
-154 \\
-166 \\
-157\end{array}$ & $\begin{array}{l}4868 \\
5264 \\
5272 \\
5261\end{array}$ & $\begin{array}{l}2839 \\
2871 \\
2879 \\
2868\end{array}$ & $\begin{array}{l}1893 \\
1914 \\
1919 \\
1912\end{array}$ & 1909 \\
\hline $\begin{array}{l}0,1256 \\
0,1251 \\
0,1403\end{array}$ & $\begin{array}{l}0,1555 \\
0,1565 \\
0,1733\end{array}$ & $\begin{array}{l}0,1886 \\
0,1881 \\
0,1902\end{array}$ & $\begin{array}{r}-12 \\
+17 \\
-\quad 4\end{array}$ & $\begin{array}{l}7,45 \\
7,43 \\
7,54\end{array}$ & $\begin{array}{l}5,02 \\
5,00 \\
5,61\end{array}$ & $\begin{array}{r}-146 \\
-146 \\
-116\end{array}$ & $\begin{array}{l}5879 \\
5867 \\
5324\end{array}$ & $\begin{array}{l}3850 \\
3838 \\
2559\end{array}$ & $\begin{array}{l}2567 \\
2559 \\
2559\end{array}$ & 2562 \\
\hline $\begin{array}{l}0,1398 \\
0,1424 \\
0,1480\end{array}$ & $\begin{array}{l}0,1723 \\
0,1723 \\
0,1794\end{array}$ & $\begin{array}{l}0,1922 \\
0,1912 \\
0,1907\end{array}$ & $\begin{array}{r}0 \\
+\quad 9 \\
+\quad 32\end{array}$ & $\begin{array}{l}7,61 \\
7,58 \\
7,57\end{array}$ & $\begin{array}{l}5,59 \\
5,70 \\
5,92\end{array}$ & $\begin{array}{r}-121 \\
-113 \\
-\quad 99\end{array}$ & $\begin{array}{l}5312 \\
5346 \\
5345\end{array}$ & $\begin{array}{l}3102 \\
3136 \\
3135\end{array}$ & $\begin{array}{l}3102 \\
3136 \\
3135\end{array}$ & 3124 \\
\hline $\begin{array}{l}0,1413 \\
0,1342 \\
0,1337\end{array}$ & $\begin{array}{c}0,1672 \\
0,1682 \\
0,1632\end{array}$ & $\begin{array}{l}0,1897 \\
0,1902 \\
0,1897\end{array}$ & $\begin{array}{r}+15 \\
-13 \\
+43\end{array}$ & $\begin{array}{l}7,52 \\
7,53 \\
7,51\end{array}$ & $\begin{array}{l}5,65 \\
5,37 \\
5,35\end{array}$ & $\begin{array}{l}-112 \\
-130 \\
-130\end{array}$ & $\begin{array}{l}5806 \\
5803 \\
5798\end{array}$ & $\begin{array}{l}\mathbf{4 7 0 7} \\
\mathbf{4 7 0 4} \\
\mathbf{4 6 9 9}\end{array}$ & $\begin{array}{l}3621 \\
3618 \\
3615\end{array}$ & 3618 \\
\hline $\begin{array}{l}0,1469 \\
0,1444 \\
0,1413\end{array}$ & $\begin{array}{l}0,1769 \\
0,1749 \\
0,1723\end{array}$ & $\begin{array}{l}0,1921 \\
0,1921 \\
0,1926\end{array}$ & $\begin{array}{l}3 \\
-16 \\
-18\end{array}$ & $\begin{array}{l}7,62 \\
7,62 \\
7,63\end{array}$ & $\begin{array}{l}5,88 \\
5,76 \\
5,65\end{array}$ & $\begin{array}{l}-104 \\
-111 \\
-119\end{array}$ & $\begin{array}{l}5536 \\
5522 \\
5555\end{array}$ & $\begin{array}{l}4437 \\
4423 \\
4456\end{array}$ & $\begin{array}{l}4437 \\
4423 \\
4456\end{array}$ & 4439 \\
\hline $\begin{array}{l}0,1479 \\
0,1520 \\
0,1500\end{array}$ & $\begin{array}{l}0,1677 \\
0,1810 \\
0,1769\end{array}$ & $\begin{array}{l}0,1921 \\
0,1916 \\
0,1916\end{array}$ & $\begin{array}{l}+30 \\
+20 \\
+33\end{array}$ & $\begin{array}{l}7,62 \\
7,61 \\
7,61\end{array}$ & $\begin{array}{l}5,92 \\
6,08 \\
6,00\end{array}$ & $\begin{array}{r}-102 \\
-\quad 92 \\
-\quad 97\end{array}$ & $\begin{array}{l}5997 \\
5699 \\
5690\end{array}$ & $\begin{array}{l}5081 \\
4600 \\
4591\end{array}$ & $\begin{array}{l}5081 \\
5111 \\
5101\end{array}$ & 5098 \\
\hline $\begin{array}{l}0,1510 \\
0,1525 \\
0,1485\end{array}$ & $\begin{array}{l}0,1738 \\
0,1718 \\
0,1703\end{array}$ & $\begin{array}{l}0,1921 \\
0,1921 \\
0,1926\end{array}$ & $\begin{array}{r}+14 \\
+10 \\
-\quad 4\end{array}$ & $\begin{array}{l}7,62 \\
7,62 \\
7,64\end{array}$ & $\begin{array}{l}6,04 \\
6,10 \\
5,94\end{array}$ & $\begin{array}{l}-95 \\
-\quad 91 \\
-102\end{array}$ & $\begin{array}{l}6090 \\
6079 \\
6080\end{array}$ & $\begin{array}{l}5357 \\
5346 \\
5347\end{array}$ & $\begin{array}{l}5639 \\
5627 \\
5628\end{array}$ & 5631 \\
\hline $\begin{array}{l}0,1543 \\
0,1568 \\
0,1523\end{array}$ & $\begin{array}{l}0,1757 \\
0,1752 \\
0,1752\end{array}$ & $\begin{array}{l}0,1915 \\
0,1915 \\
0,1925\end{array}$ & $\begin{array}{l}-\quad 6 \\
+\quad 4 \\
-\quad 6\end{array}$ & $\begin{array}{l}7,61 \\
7,61 \\
7,64\end{array}$ & $\begin{array}{l}6,17 \\
6,27 \\
6,09\end{array}$ & $\begin{array}{l}-\quad 86 \\
-\quad 80 \\
-\quad 93\end{array}$ & $\begin{array}{l}6200 \\
6203 \\
6195\end{array}$ & $\begin{array}{l}5467 \\
5470 \\
5462\end{array}$ & $\begin{array}{l}6074 \\
6078 \\
6069\end{array}$ & 6074 \\
\hline $\begin{array}{l}0,1572 \\
0,1489 \\
0,1509\end{array}$ & $\begin{array}{l}0,1776 \\
0,1728 \\
0,1766\end{array}$ & $\begin{array}{l}0,1908 \\
0,1898 \\
0,1913\end{array}$ & $\begin{array}{r}-9 \\
+26 \\
+22\end{array}$ & $\begin{array}{l}7,58 \\
7,53 \\
7,60\end{array}$ & $\begin{array}{l}6,29 \\
5,96 \\
6,04\end{array}$ & $\begin{array}{l}-77 \\
-\quad 94 \\
-\quad 94\end{array}$ & $\begin{array}{l}5384 \\
5373 \\
5457\end{array}$ & $\begin{array}{l}3919 \\
3908 \\
3259\end{array}$ & $\begin{array}{l}3266 \\
3257 \\
3259\end{array}$ & 3261 \\
\hline $\begin{array}{l}0,1377 \\
0,1543 \\
0,1538\end{array}$ & $\begin{array}{l}0,1698 \\
0,1776 \\
0,1776\end{array}$ & $\begin{array}{c}0,1903 \\
0,1893 \\
0,1893\end{array}$ & $\begin{array}{lr}+ & 1 \\
+ & 7 \\
- & 24\end{array}$ & $\begin{array}{l}7,54 \\
7,52 \\
7,52\end{array}$ & $\begin{array}{l}\breve{5}, 51 \\
6,17 \\
6,15\end{array}$ & $\begin{array}{r}-122 \\
-\quad 81 \\
-\quad 82\end{array}$ & $\begin{array}{l}5496 \\
5896 \\
5896\end{array}$ & $\begin{array}{l}3286 \\
3686 \\
3686\end{array}$ & $\begin{array}{l}2833 \\
2835 \\
2835\end{array}$ & 2834 \\
\hline
\end{tabular}


418 Stohmann: Calorimetrische Untersuchnngen.

\begin{tabular}{|c|c|c|c|c|}
\hline & & $\begin{array}{l}\text { Molekula } \\
\text { gewicht }\end{array}$ & $\begin{array}{c}\text { - Wärmewerth } \\
\text { cal. }\end{array}$ & $\begin{array}{l}\text { Differenz } \\
\text { cal. }\end{array}$ \\
\hline Essigsäure & $\mathrm{O}, \mathrm{H}, \mathrm{O}$ & 60 & 213293 & \\
\hline Propionsänre & $\mathrm{C}_{3} \mathrm{H}_{6} \mathrm{O}_{2}$ & 74 & 367945 & 154652 \\
\hline Buttersäure & $\mathrm{C}_{4} \mathrm{H}_{8} \mathrm{O}_{2}$ & 88 & 522678 & 15300 \\
\hline Valeriansäure & $\mathrm{O}_{5} \mathrm{H}_{\mathrm{in}} \mathrm{O}_{2}$ & 102 & 676676 & 153998 \\
\hline Capronsäure & $\mathrm{C}_{6} \mathrm{H}_{12} \mathrm{O}_{2}$ & 116 & 831246 & 154570 \\
\hline Caprylsäure & $\mathrm{O}_{g} \mathrm{H}_{1 f} \mathrm{O}_{2}$ & 144 & 1139965 & $\begin{array}{l}104309 \times 2 \\
104748 \times 2\end{array}$ \\
\hline Caprinsüure & $\mathrm{C}_{10} \mathrm{H}_{30} \mathrm{O}_{2}$ & 172 & 1449462 & 1.04840 \\
\hline Laurinsäure & $\mathrm{O}_{12} \mathrm{H}_{24} \mathrm{O}_{2}$ & 200 & 1757181 & $\begin{array}{l}103809 \times \\
153709 \times\end{array}$ \\
\hline Myristinsäure & & 228 & 2064598 & \\
\hline
\end{tabular}

Zur Controlle der ganzen Zahlenreihe sind die hier ermittelten Werthe, soweit es thunlich ist, mit den durch Verbrennung der freien Säuren gefundenen Werthen zu vergleichen.

Wärmewerth

aus dem Silbersalz Freie Săure

$\begin{array}{lrrr}\text { Capronsäure, flüssig } & 831246 & 830212=100: 99,9 \\ \text { Caprinsäure " } & 1449462 & 1455636=100: 100,4 \\ \text { Laurinsäure " } & 1757181 & 1756338=100: 99,9 \\ \text { Myristinsäure " } & 2064598 & 2064649=100: 100,0\end{array}$

Und benutzen wir die als Mittel aller Differenzen gefundene Zahl 154275, um von der festen Laurinsäure aufwärts steigend die Werthe für die übrigen festen Säuren zu berechnen, so kommen wir zu folgenden Werthen:

\section{Berechnet Gefunden}

Laurinsäure, fest $\mathrm{C}_{12} \mathrm{H}_{2,} \mathrm{O}_{2}$ 1747600

Myristinsäure " $\mathrm{O}_{14} \mathrm{H}_{28} \mathrm{O}_{2} 20561502053824=100: 99,9$ Palmitinsäure " $\mathrm{C}_{18} \mathrm{H}_{32} \mathrm{O}_{2} 23647002361856=100: 99,9$ Stearinsäure $\quad \mathrm{O}_{18} \mathrm{H}_{36} \mathrm{O}_{2} 26732502677834=100: 100,2$

Die grosse Uebereinstimmung dieser Zahlen liefort den Beweis für die Zulässigkeit der von uns angewandten Methode der Ableitung der Werthe. Bei all den Säuren, für welche dirccte Bestimmungen des Wärmewerthes vorliegen, nähern sich die aus den Silbersalzen abgeleiteten Zahlen noch mehr den gefundenen Verbrennungswärmen der 
Stohmann: Calorimetrische Untersuchungen. 419 freien Säuren, als dies bei unseren früheren Rechnungen der Fall war. Andererseits wird durch die vollkommene Gleichmässigkeit, welche sich in den Differenzen der einzelnen Glieder der ganzen Reihe der flüssigen Säuren zeigt, der Nachweis geliefert, dass die Verbrennungswärmen von der Essigsäure bis zur Valeriansäure ebenso wenig mit Fehlern behaftet sind, wie die der kohlenstoffreicheren Säuren, deren Werthe wir direct controlliren konnten. Es wird demnach auch der Wärmewerth der A meiscusäure, für welche wir keine Bestimmung ausführen konnten, weil das ameisensaure Silber sich beim Erwärmen mit lebhafter Explosion zersetzt, sich von dem der Essigsäure um den Betrag von 154275 cal. unterscheiden, und es kann derselbe zu 59018 cal. angenommen werden.

Nach Berthelot ${ }^{1}$ ) ist die Bildungswärme der Ameisensäure

$\mathrm{C}$ (Diamant) $+\mathrm{H}_{2}+\mathrm{O}_{2}=\mathrm{CH}_{2} \mathrm{O}_{2}$ (flüssig) +93000 cal.

Hieraus leitet sich die Verbrennungswärme 70000 cal. ab.

Für die Essigsäure giebt Berthelot ${ }^{2}$ ) den Werth 199400 cal. als Verbrennungswärme. Hiernach würde die Differenz zwischen den beiden homologen Säuren nur 129400 cal. betragen. Da aber eine so geringe Differenz zwischen zwei homologen Verbindungen noch niemals beobachtet worden ist, so muss die Richtigkeit dieser Zahlen bezweifelt werden.

Die glänzendste Bestätigung für die Richtigkeit der von uns abgeleiteten Zahl für den Wärmewerth der Ameisensäure und damit für den der ganzen Reihe der homologen Säuren ergiebt sich aus der Bestimmung von Thomsen ${ }^{3}$ ), welcher die Verbrennungswärme dieser Säure auf nassem Wege, durch Oxydation mit übermangansauren Kalium, bestimmte und für dieselbe 60193 cal. fand. Beide auf gänzlich verschiedenem Wege gewonnenen Zahlen decken einander vollständig, und hiermit ist der sichere Beweis erbracht, dass die ganze Reihe der homologen Säuren, von der Ameisen-

1) Ann. Chim. Phys. [5] 5, 317.

9) Das. [5] 23, 200.

3) Ber. Berl. chem. Ges. b, 957. 
420 Stohmann: Calorimetrische Untersuchnngen.

säure bis zur Stearinsäure hin, und höchst wahrscheinlich über dieselbe hinaus, eine gleichmässig verlaufende Kette bildet, deren einzelne Glieder, bei gleichem Aggregatzustand, sich in ihrem Wärmewerth um die unveränderliche Grösse von 154275 cal. von einander unterscheiden.

\section{Fünfte Abhandlung.}

Ueber die thermochemischen Beziehungen der Säuren und Alkohole der Fettsäurereihe;

von

\section{F. Stohmann.}

Da beim Uebergange der normalen Alkohole in die Säuren der Fettsäurercihe ein einfacher Austausch von 2wei Wasserstoffatomen der $\mathrm{CH}_{2} \mathrm{OH}$ Gruppe gegen ein Sauerstoffatom, unter Bildung der Carboxylgruppe stattfindet, so muss dieser, bei allen Gliedern der homologen Reihe sich wiederholende Process mit einer bestimmten Wärmetönung verbunden sein, deren Grösse sich aus der Diflerenz der Verbrennungswärmen der Alkohole und der Säuren ergiebt. Nachdem durch die vorstehende Untersuchung die Wärmewerthe der ganzen Reihe der fetten Säuren festgestellt waren, lag es nahe, die zwischen den Säuren und Alkoholen obwaltenden Beziehungen näher zu untersuchen, um so mehr, da in neuerer Zeit die Verbrennungswärme einer Reihe von Alkoholen ermittelt worden ist. Als Material benutzen wir für:

Methylalkohol, die Bestimmung von Favre und Silbermann. ${ }^{2}$ ) Die für den Methylalkohol angenommene Zahl kann auf grosse Zuverlässigkeit keinen Anspruch machen, da sie aus vier Verbrennungen abgelcitet ist, deren Einzel-

1) Anu. Chim. Phys. [3] 34, 434. 